\title{
Conventional type 1 dendritic cells in protective antitumor immunity and its potential in hepatocellular carcinoma
}

\author{
Chunfeng Qu, Kun Chen, Shao-Yan Cheng \\ State Key Lab of Molecular Oncology \& Immunology Department, National Cancer Center/National Clinical Research Center \\ for Cancer/Cancer Hospital, Chinese Academy of Medical Sciences and Peking Union Medical College, Beijing 100021, China.
}

Correspondence to: Prof. Chunfeng Qu, State Key Lab of Molecular Oncology, National Cancer Center/National Clinical Research Center for Cancer/Cancer Hospital, Chinese Academy of Medical Sciences and Peking Union Medical College, No. 17 Panjiayuan South lane, Chaoyang District, Beijing 100021, China. E-mail: quchf@cicams.ac.cn

How to cite this article: Qu C, Chen K, Cheng SY. Conventional type 1 dendritic cells in protective antitumor immunity and its potential in hepatocellular carcinoma. Hepatoma Res 2020;6:38. http://dx.doi.org/10.20517/2394-5079.2020.12

Received: 6 Feb 2020 First Decision: 18 Mar 2020 Revised: 27 Apr 2020 Accepted: 8 Jun 2020 Published: 10 Jul 2020

Academic Editor: Ying-Hong Shi Copy Editor: Cai-Hong Wang Production Editor: Jing Yu

\begin{abstract}
Immunotherapy is revolutionizing the clinical management of cancer patients by modulating $T$ cells and natural killer cells. Dendritic cells (DCs) have the capacity to orchestrate the expansion and function of these effector cells both in lymphoid and non-lymphoid tissues of cancer patients. Distinct subtypes of DCs have various capacities to prime and activate different $T$ cell responses. Here, we review conventional type 1 dendritic cells (CDC1s) and their crucial role in protective anti-tumor immunity. Targeting $\mathrm{CDC}$ 1s as a cancer vaccine against the development of hepatocellular carcinoma will be discussed.
\end{abstract}

Keywords: Conventional type 1 dendritic cells, antitumor immunity, hepatocellular carcinoma, cancer vaccine

\section{INTRODUCTION}

Immunotherapy is now widely considered as an important tool for the treatment of individuals with cancer. Several effective immunotherapy approaches have been developed over the past decade, including adoptive cell transfer (e.g., CAR-T therapy) and immune checkpoint blockade (e.g., anti-PD1/PDL1 antibodies $)^{[1,2]}$. In solid tumors including hepatocellular carcinoma (HCC), the tumor microenvironment contains a large amount of stromal cells and immune cells, which shape cancer development and impact

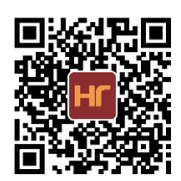


upon the response to tumor therapy. The effectiveness of immune-based therapeutic strategies clearly demonstrates the possibility of eradicating cancer through cellular immunity, particularly when active $\mathrm{T}$ cells recognize cognate tumor antigens ${ }^{[1,3]}$. Dendritic cells (DCs) have the capacity to prime naive $\mathrm{T}$ cells by inducing functional polarization, and are in charge of orchestrating the expansion and functions of $\mathrm{T}$ cells and natural killer (NK) cells in lymphoid and non-lymphoid tissues of cancer patients. About 25 years ago, some investigators put forward the concept of harnessing DC immunogenicity to induce protective responses in cancer patients ${ }^{[4,5]}$. Nevertheless, results have been far below expectations. These failures occurred due to the almost exclusive usage of monocyte-derived cells (MoDCs) and tumor-associated antigens. The advancement in basic understanding of the heterogeneity and functional plasticity of different DC subsets suggests that conventional (classical) type 1 DCs (cDC1s) other than MoDCs may be better suited for this purpose.

\section{DENDRITIC CELL SUBSETS}

Shortly after the discovery of DCs by Steinman in 1973, van Furth grouped them under the mononuclear phagocyte system. Since then monocytes, macrophages and DCs have been grouped together, and they are distinguished on the basis of their morphology, function and origin ${ }^{[6]}$. However, lineage-tracing studies by different groups demonstrate that most macrophages in adults are maintained independently of blood monocytes and rely on self-renewal of embryonically derived macrophages under steady-state conditions $^{[7,8]}$. The circulating monocytes could contribute to the expanding pool of macrophages in the liver under inflammation status ${ }^{[9,10]}$. DCs arise from a common DC precursor in the bone marrow, and their development depends on the cytokine Flt3 $\mathrm{L}^{[11,12]}$. Although monocytes in some inflammation develop to inflammatory DCs, termed IDCs ${ }^{[13]}$, DCs are now generally grouped into conventional (classical) type 1 DCs (cDC1s), conventional type 2 DCs (cDC2s) and plasmacytoid DCs (pDCs) ${ }^{[14]}$.

In mice, $\mathrm{cDC} 1 \mathrm{~s}$ include murine lymphoid $\mathrm{CD} 8 \alpha^{+} \mathrm{DCs}$, migratory $\mathrm{CD} 103^{\text {high }} \mathrm{DCs}$, and dermal $\mathrm{CD} 207^{+} \mathrm{DCs}$; cDC2s include lymphoid $\mathrm{CD}_{4}{ }^{+} \mathrm{DCs}$ and migratory CD11 $\mathrm{b}^{+} \mathrm{DCs}$; pDCs are IFN-producing DCs with the cell surface markers B220, PDCA.1 and Ly6C $\mathrm{C}^{[14]}$. DCs have been divided into many different subsets based on the expression of surface markers including $\mathrm{CD} 40, \mathrm{CD} 11 \mathrm{c}, \mathrm{CD} 103$ (integrin $\alpha \mathrm{E}$ ), $\mathrm{CD} 11 \mathrm{~b}$ (integrin $\alpha \mathrm{M}$ ), F4/80, CD8 $\alpha, C D 24, C D 172 a$ (SIRP $\alpha$ and SHPS1), $\mathrm{CX}_{3} \mathrm{C}$-chemokine receptor 1 (CX ${ }_{3} \mathrm{CR} 1$ ), XC-chemokine receptor 1 (XCR1), CLEC9A (DNGR1), E-cadherin (cadherin 1) and CD64 (FcrRI) ${ }^{[14]}$. Inflammation further complicates the picture as mononuclear phagocytes in inflamed tissues undergo phenotypical changes. Some researchers consider monocyte-derived cells as MoDCs or macrophages, based on CD11c expression $^{[13-15]}$. The analysis of gene expression profiles revealed that the gene transcripts in different populations of macrophages are diverse, and some mRNA transcripts and surface proteins were selectively expressed by macrophages but not DCs ${ }^{[16]}$. Therefore, macrophages, monocytes and DCs are different cell types with distinct ontogeny ${ }^{[7-14,16]}$.

\section{DC-BASED CANCER VACCINES}

DC-based cancer vaccines typically culture DCs with various tumor associated antigens ex vivo, such as pulsing with peptides, whole proteins, tumor lysates, or fusion of DCs with entire tumor cells ${ }^{[1,4,5]}$. Most DCs were generated from peripheral blood monocular cells (PBMCs)-derived CD $14^{+}$monocytes or CD $34^{+}$ hematopoietic stem and progenitor cells via culturing with granulocyte-macrophage colony-stimulating factor (GM-CSF) and interleukin 4 (IL-4). The efficacy of such vaccine formulations is suboptimal since the macrophages were generated in culture with GM-CSF and its migratory capacity limited to lymph nodes ${ }^{[1,5]}$.

To overcome these tedious processes and the uncertainty of quality, approaches that home antigens directly to DCs in vivo via DC receptors were developed, such as antibodies against DEC-205, Clec9A and $\mathrm{Clec} 12 \mathrm{~A}^{[1,4,17,18]}$. By using model antigens, i.e., OVA, or antigens from microbial products, direct targeting 
strategies showed some protective effects on tumor growth ${ }^{[4,18,19]}$. However, these effects were suboptimal in clinical trials when the antibody against DEC-205, which is expressed in different subsets of DCs in human, was conjugated with the tumor antigen NY-ESO ${ }^{[20]}$. Therefore, when contemplating DCs for in vivo targeting for tumor vaccination, it is important to consider the differences of targeting DC receptors and their function ${ }^{[1,5]}$.

\section{THE CDC1S AND THEIR ROLES AGAINST CANCERS}

The "cross-presentation" by DCs of tumor antigens that are expressed in solid tumors is crucial for generating effective $\mathrm{CD}^{+}$cytotoxic $\mathrm{T}$ lymphocytes (CTLs) ${ }^{[1,21]}$. Accumulated experimental evidence revealed that distinct subtypes of DCs show various capacities to prime and activate different $\mathrm{T}$ cells ${ }^{[1,5]}$. Mouse $\mathrm{cDC} 1 \mathrm{~s}$ were identified as the most efficient cells in cross-presenting cellular-associated antigens to initiate $\mathrm{CD}^{+} \mathrm{T}$ cells ${ }^{[22-25]}$. The following transcription factors $\operatorname{ICSBP}\left(\operatorname{Irf} f 8^{[26]}, \operatorname{Id}^{\left[{ }^{[27]}\right.}, \operatorname{Batf}^{[28]}, \mathrm{Nfil}^{[29]}\right.$ were reported to control the development of $\mathrm{CDC} 1 \mathrm{~s}$. Further epistasis analysis after single-cell RNA sequencing revealed a genetic hierarchy. The Nfil3 induces a transition from common DC precursors to express high levels of $I d 2$ and low levels of Zeb2. Upon Id2 induction, Batf3 expression is increased and Zeb2 is repressed. Meanwhile, $I d 2$ extinguished E-protein activity at the $+41-\mathrm{kb} \operatorname{Irf} 8$ enhancer, and the expressed BATF3 acted at the $+32-\mathrm{kb} \operatorname{Irf} 8$ enhancer to maintain $\operatorname{Irf} 8$ activation for the commitment of cDC1 clonogenic progenitors ${ }^{[30,31]}$. Currently, the homolog and functional equivalent of mouse $\mathrm{CDC} 1 \mathrm{~s}$ have been identified as CD141 $\left(\mathrm{BDCA}_{3}\right)^{+} \mathrm{XCR}^{+}{ }^{+} \mathrm{DCs}$ in humans ${ }^{[14,32,33]}$. Both mouse and human $\mathrm{CDC} 1 \mathrm{~s}$ selectively express the chemokine receptor XCR1 and C-type lectin endocytic receptor CLEC9 $\mathrm{A}^{[4,14,34,35]}$.

The role of cDC1s in antitumor immunity was investigated in different animal models. In the Batf3-/mouse, the rejection of highly immunogenic syngeneic tumors was impaired ${ }^{[28]}$. Using 2-photon intravital imaging, the spatial organization of DCs and macrophages within the tumor and their dynamics with T cells were analyzed. It was found that macrophage populations were preferentially marginating tightly on tumoral lesions, DC populations were typically in separate collagen-rich zones distal to tumor lesions. Stable $\mathrm{T}$ cell interactions were largely confined to tumor margins, where macrophage populations dominated and DC numbers were little ${ }^{[36]}$. Analyzing the different types of human tumors in TCGA data revealed that patients with a high ratio of the $\mathrm{CD}_{103^{+}} / \mathrm{CD}_{103}$ gene showed better survival compared to those with a low ratio ${ }^{[37]}$. In mouse melanoma models, it was found that $\mathrm{CD}_{103^{+}}{ }^{\mathrm{DC}}$ s were the only antigen-presenting cells to transport intact antigens to lymph nodes for priming tumor-specific $\mathrm{CD}^{+}$ $\mathrm{T}$ cells. When $\mathrm{CD} 103^{+} \mathrm{DC}$ progenitors in the tumor were expanded and activated, the effects of anticheckpoint inhibitor were enhanced for anti-tumor responses ${ }^{[38]}$.

In the context of cancer, there are many different cell populations. In addition to cDC1s, several myeloid cell populations, including tumor-associated macrophages, are also able to acquire tumor material. However, cDC1s have been demonstrated to be superior to other cells in stimulating $\mathrm{T}$ cell activation and proliferation ${ }^{[36-38]}$. The stimulatory function of $\mathrm{cDC} 1 \mathrm{~s}$ in tumors is not restricted to T cells only. IL-12 production by these cells support IFN- $\gamma$ production from NK cells for eradicating established tumor cells $^{[39,40]}$. More importantly, activated NK cells can generate the chemokines CCL5 and XCL1 to recruit cDC1s into the tumor microenvironment and promote cancer immune control ${ }^{[25]}$. In melanoma patients, it was found that the abundance of $\mathrm{cDC1s}$ is associated with intra-tumor gene expression of the cytokine FLT3LG, which is predominantly produced by NK cells in tumors. The numbers of $\mathrm{CDC} 1$ is correlated with NK frequency and the patients' response to anti-PD1 immunotherapy ${ }^{[41]}$. It was found that $\mathrm{CDC} 1 \mathrm{~s}$ might be the main source of CXCL9 and CXCL10, and these chemokines are able to recruit CXCR3 ${ }^{+}$effector T cells ${ }^{[24,40,42,43]}$. Therefore, the $\mathrm{CDC} 1 \mathrm{~s}$ in tumors and their interactions with NK and effector T cells establish local anti-tumor immunity to control and eventually, eradicate established tumors ${ }^{[24,40]}$. 


\section{TARGETING CDC1S FOR HCC INTERVENTION}

Some patients with a variety of cancers, including HCC, benefit from immune checkpoint inhibitors ${ }^{[44,45]}$. Since not all HCC patients are sensitive to this therapy, research has found that WNT activation correlated with $\mathrm{T}$ cell exclusion and resistance to anti-PD1 therapy ${ }^{[46,47]}$. By using a murine autochthonous liver cancer model based on hydrodynamic tail vein delivery of different genetic elements, it was found that WNT activation led to defective DC recruitment, mainly $\mathrm{cDC} 1 \mathrm{~s}$. As a consequence, the anti-tumor immune response was impaired. The impaired immunity in HCC from WNT activation might be due to decreased CCL5 secretion by the tumor ${ }^{[48]}$. These investigations pointed to the importance of $\mathrm{CDC} 1$ recruitment in HCC immunotherapy.

HCCs arising in cirrhosis are usually preceded by the appearance of malignant precancerous nodules ${ }^{[49]}$. The liver of a cirrhotic patient may harbor either a single, benign or precancerous malignant nodule, or even both. HCC progression could be interfered with if the malignant progenitors in cirrhosis were eliminated. At this stage, it is difficult to treat with conventional means such as surgery, radiation, and chemotherapy. However, it may still be controllable by stimulating the immune response through cancer vaccines. Tumor-associated antigens, which are re-expressed in tumor and not/lowly expressed in normal tissues, might potentially be appropriate targets ${ }^{[50]}$. Previous studies have documented that inducing the generation of specific T cells to alpha-fetoprotein (AFP), which is re-expressed in most HCCs, led to tumor regression ${ }^{[50]}$ and prevented carcinogen-induced murine autochthonous HCC $^{[51]}$. However, in regenerating mouse liver significant hepatocyte damage was observed ${ }^{[52]}$. Two decades ago, glypican-3 (GPC3) was identified as a new HCC-associated antige ${ }^{[53]}$. Different from AFP, it is undetectable in cirrhotic livers, or even in benign hepatic lesions. Tissue expression of GPC3 was used to discriminate the nature of $\mathrm{a}<2 \mathrm{~cm}$ hepatocellular lesion lacking HCC radiological features detected in a cirrhotic patient under surveillance. Up to $60 \%$ of early HCCs showed immunoreactivity to GPC3, either as membranous and/or cytoplasmic staining in biopsy material ${ }^{[53]}$. We therefore hypothesized that eliciting the host's own specific $\mathrm{T}$ cell immunity against GPC3 could interfere with disease progression in cirrhosis patients.

With the growing importance of $\mathrm{cDC} 1 \mathrm{~s}$ in initiating effective anti-tumor immunity, and the selective expression of XCR1 on these $\mathrm{CDC} 1 \mathrm{~s}$, the XCL1-GPC3 fusion protein may be an efficient cancer vaccine. We have linked the XCL1 chemokine to GPC3 for in vivo targeting to induce GPC3-specific CTLs for eliminating GPC3-positive HCC. Our results showed that the expressed XCL1-GPC3 chemoattracted murine $\mathrm{XCR} 1{ }^{+} \mathrm{CD} 8 \alpha^{+} \mathrm{DCs}$ and human $\mathrm{XCR} 1^{+} \mathrm{CD} 141^{+} \mathrm{DCs}$ in vitro and promoted IL-12 production. After the $m X C L 1-G P C 3$ plasmid was injected subcutaneously, the expressed mXCL1-GPC3 protein was detected mainly in CD $8 \alpha^{+}$DCs of mice draining lymph nodes. XCL1-GPC3-targeted DCs enhanced antigen-specific $\mathrm{CD}^{+} \mathrm{T}$ cell proliferation and induced the de novo generation of GPC3-specific $\mathrm{CD} 8^{+} \mathrm{T}$ cells, which abolished GPC3-expressing tumor cells both in the murine and human systems. In a murine autochthonous liver cancer model of AlBlHBV mice, the 3-dose (30- $\mu \mathrm{g}$ in total) immunization suspended tumor development with significantly reduced tumor incidence and tumor load compared with GPC3immunized mice. Notably, the mouse livers have increased infiltration of GPC3-specific CD ${ }^{+} \mathrm{T}$ cells, activated NK cells and NKT cells after $m X C L 1-G P C 3$ immunization. The antitumor effects of these cells were further enhanced by the administration of anti-PD- ${ }^{[40]}$. Therefore, the XCL1-GPC3, which targets cDC1s, might be a promising cancer vaccine to compensate for the deficiency of checkpoint blockades in HCC immunotherapy.

\section{SUMMARY}

The presence of cDC1s in the tumor microenvironment is often associated with a good prognosis in cancer patients and better response to immune checkpoint inhibitors. This cell population is an attractive target for delivering HCC tumor antigens to induce antitumor specific T cell responses. The combination of cDC1- 
targeting cancer vaccines and checkpoint inhibitors will improve the efficacy of immunotherapy. Murine models have proven the effect of HCC cancer vaccines in compensating for anti-PD-1 by targeting cDC1s. However, clinical trials of such cancer vaccine are still required. It is also necessary to understand how cDC1s regulate anti-tumor immunity.

\section{DECLARATIONS}

\section{Authors' contributions}

Manuscript draft and finalization: Qu C

Participated in drafting of the manuscript: Chen K, Cheng SY

\section{Availability of data and materials}

Not applicable.

\section{Financial support and sponsorship}

This work was supported by the Natural Science Foundation of China (No. 81571620) and the Chinese Academy of Medical Sciences Innovative Medicine (No. 2016-I2M-1-007).

\section{Conflicts of interest}

All authors declared that there are no conflicts of interest.

\section{Ethical approval and consent to participate}

Not applicable.

\section{Consent for publication}

Not applicable.

\section{Copyright}

(c) The Author(s) 2020.

\section{REFERENCES}

1. Chen DS, Mellman I. Elements of cancer immunity and the cancer-immune set point. Nature 2017;541:321-30.

2. Friedrich MJ. Immunotherapy 2.0: improving the response to checkpoint inhibitors. JAMA 2019;321:131-3.

3. Shankaran V, Ikeda H, Bruce AT, White JM, Swanson PE, et al. IFNgamma and lymphocytes prevent primary tumour development and shape tumour immunogenicity. Nature 2001;410:1107-11.

4. van Dinther D, Stolk DA, van de Ven R, van Kooyk Y, de Gruijl TD, et al. Targeting C-type lectin receptors: a high-carbohydrate diet for dendritic cells to improve cancer vaccines. J Leukoc Biol 2017;102:1017-34.

5. Romero P, Banchereau J, Bhardwaj N, Cockett M, Disis ML, et al. The Human Vaccines Project: A roadmap for cancer vaccine development. Sci Transl Med 2016;8:334ps9.

6. Muller WA, Randolph GJ. Migration of leukocytes across endothelium and beyond: molecules involved in the transmigration and fate of monocytes. J Leukoc Biol 1999;66:698-704.

7. Jakubzick C, Gautier EL, Gibbings SL, Sojka DK, Schlitzer A, et al. Minimal differentiation of classical monocytes as they survey steady-state tissues and transport antigen to lymph nodes. Immunity 2013;39:599-610.

8. Yona S, Kim KW, Wolf Y, Mildner A, Varol D, et al. Fate mapping reveals origins and dynamics of monocytes and tissue macrophages under homeostasis. Immunity 2013;38:79-91.

9. Scott CL, Zheng F, De Baetselier P, Martens L, Saeys Y, et al. Bone marrow-derived monocytes give rise to self-renewing and fully differentiated Kupffer cells. Nat Commun 2016;7:10321.

10. Ramachandran P, Dobie R, Wilson-Kanamori JR, Dora EF, Henderson BEP, et al. Resolving the fibrotic niche of human liver cirrhosis at single-cell level. Nature 2019;575:512-8.

11. Naik SH, Sathe P, Park HY, Metcalf D, Proietto AI, et al. Development of plasmacytoid and conventional dendritic cell subtypes from single precursor cells derived in vitro and in vivo. Nat Immunol 2007;8:1217-26.

12. Onai N, Obata-Onai A, Schmid MA, Ohteki T, Jarrossay D, et al. Identification of clonogenic common Flt3+M-CSFR+ plasmacytoid and conventional dendritic cell progenitors in mouse bone marrow. Nat Immunol 2007;8:1207-16.

13. Palomino-Segura M, Perez L, Farsakoglu Y, Virgilio T, Latino I, et al. Protection against influenza infection requires early recognition by 
inflammatory dendritic cells through C-type lectin receptor SIGN-R1. Nat Microbiol 2019;4:1930-40.

14. Guilliams M, Ginhoux F, Jakubzick C, Naik SH, Onai N, et al. Dendritic cells, monocytes and macrophages: a unified nomenclature based on ontogeny. Nat Rev Immunol 2014;14:571-8.

15. Menezes S, Melandri D, Anselmi G, Perchet T, Loschko J, et al. The Heterogeneity of Ly6Chi Monocytes Controls Their Differentiation into iNOS+ Macrophages or Monocyte-Derived Dendritic Cells. Immunity 2016;45:1205-18.

16. Gautier EL, Shay T, Miller J, Greter M, Jakubzick C, et al; Immunological Genome Consortium. Gene-expression profiles and transcriptional regulatory pathways that underlie the identity and diversity of mouse tissue macrophages. Nat Immunol 2012;13:1118-28.

17. Caminschi I, Proietto AI, Ahmet F, Kitsoulis S, Shin Teh J, et al. The dendritic cell subtype-restricted C-type lectin Clec9A is a target for vaccine enhancement. Blood 2008;112:3264-73.

18. Bonifaz LC, Bonnyay DP, Charalambous A, Darguste DI, Fujii S, et al. In vivo targeting of antigens to maturing dendritic cells via the DEC-205 receptor improves T cell vaccination. J Exp Med 2004;199:815-24.

19. Neubert K, Lehmann CH, Heger L, Baranska A, Staedtler AM, et al. Antigen delivery to CD11c+CD8- dendritic cells induces protective immune responses against experimental melanoma in mice in vivo. J Immunol 2014;192:5830-8.

20. Dhodapkar MV, Sznol M, Zhao B, Wang D, Carvajal RD, et al. Induction of antigen-specific immunity with a vaccine targeting NYESO-1 to the dendritic cell receptor DEC-205. Sci Transl Med 2014;6:232ra51.

21. Cruz FM, Colbert JD, Merino E, Kriegsman BA, Rock KL. The Biology and Underlying Mechanisms of Cross-Presentation of Exogenous Antigens on MHC-I Molecules. Annu Rev Immunol 2017;35:149-76.

22. den Haan JM, Lehar SM, Bevan MJ. CD8(+) but not CD8(-) dendritic cells cross-prime cytotoxic T cells in vivo. J Exp Med 2000;192:1685-96.

23. Kitano M, Yamazaki C, Takumi A, Ikeno T, Hemmi H, et al. Imaging of the cross-presenting dendritic cell subsets in the skin-draining lymph node. Proc Natl Acad Sci U S A 2016;113:1044-9.

24. Cancel JC, Crozat K, Dalod M, Mattiuz R. Are Conventional Type 1 Dendritic Cells Critical for Protective Antitumor Immunity and How? Front Immunol 2019;10:9.

25. Böttcher JP, Bonavita E, Chakravarty P, Blees H, Cabeza-Cabrerizo M, et al. NK Cells Stimulate Recruitment of cDC1 into the Tumor Microenvironment Promoting Cancer Immune Control. Cell 2018;172:1022-1037.e14.

26. Schiavoni G, Mattei F, Sestili P, Borghi P, Venditti M, et al. ICSBP is essential for the development of mouse type I interferon-producing cells and for the generation and activation of CD8alpha(+) dendritic cells. J Exp Med 2002;196:1415-25.

27. Hacker C, Kirsch RD, Ju XS, Hieronymus T, Gust TC, et al. Transcriptional profiling identifies Id 2 function in dendritic cell development. Nat Immunol 2003;4:380-6.

28. Hildner K, Edelson BT, Purtha WE, Diamond M, Matsushita H, et al. Batf3 deficiency reveals a critical role for CD8alpha+ dendritic cells in cytotoxic T cell immunity. Science 2008;322:1097-100.

29. Kashiwada M, Pham NL, Pewe LL, Harty JT, Rothman PB. NFIL3/E4BP4 is a key transcription factor for CD $8 \alpha^{+}$dendritic cell development. Blood 2011;117:6193-7.

30. Bagadia P, Huang X, Liu TT, Durai V, Grajales-Reyes GE, et al. An Nfil3-Zeb2-Id2 pathway imposes Irf8 enhancer switching during cDC1 development. Nat Immunol 2019;20:1174-85.

31. Grajales-Reyes GE, Iwata A, Albring J, Wu X, Tussiwand R, et al. Batf3 maintains autoactivation of Irf8 for commitment of a CD8 $\alpha(+)$ conventional DC clonogenic progenitor. Nat Immunol 2015;16:708-17.

32. Poulin LF, Salio M, Griessinger E, Anjos-Afonso F, Craciun L, et al. Characterization of human DNGR-1+ BDCA3+ leukocytes as putative equivalents of mouse CD8alpha+ dendritic cells. J Exp Med 2010;207:1261-71.

33. Jongbloed SL, Kassianos AJ, McDonald KJ, Clark GJ, Ju X, et al. Human CD141+ (BDCA-3)+ dendritic cells (DCs) represent a unique myeloid DC subset that cross-presents necrotic cell antigens. J Exp Med 2010;207:1247-60.

34. Dorner BG, Dorner MB, Zhou X, Opitz C, Mora A, et al. Selective expression of the chemokine receptor XCR1 on cross-presenting dendritic cells determines cooperation with CD8+ T cells. Immunity 2009;31:823-33.

35. Crozat K, Guiton R, Contreras V, Feuillet V, Dutertre CA, et al. The XC chemokine receptor 1 is a conserved selective marker of mammalian cells homologous to mouse CD8alpha+ dendritic cells. J Exp Med 2010;207:1283-92.

36. Engelhardt JJ, Boldajipour B, Beemiller P, Pandurangi P, Sorensen C, et al. Marginating dendritic cells of the tumor microenvironment cross-present tumor antigens and stably engage tumor-specific T cells. Cancer Cell 2012;21:402-17.

37. Broz ML, Binnewies M, Boldajipour B, Nelson AE, Pollack JL, et al. Dissecting the tumor myeloid compartment reveals rare activating antigen-presenting cells critical for T cell immunity. Cancer Cell 2014;26:638-52.

38. Salmon H, Idoyaga J, Rahman A, Leboeuf M, Remark R, et al. Expansion and Activation of CD103(+) Dendritic Cell Progenitors at the Tumor Site Enhances Tumor Responses to Therapeutic PD-L1 and BRAF Inhibition. Immunity 2016;44:924-38.

39. Mittal D, Vijayan D, Putz EM, Aguilera AR, Markey KA, et al. Interleukin-12 from CD103+ Batf3-Dependent Dendritic Cells Required for NK-Cell Suppression of Metastasis. Cancer Immunol Res 2017;5:1098-108.

40. Chen K, Wu Z, Zhao H, Wang Y, Ge Y, et al. XCL1/Glypican-3 Fusion Gene Immunization Generates Potent Antitumor Cellular Immunity and Enhances Anti-PD-1 Efficacy. Cancer Immunol Res 2020;8:81-93.

41. Barry KC, Hsu J, Broz ML, Cueto FJ, Binnewies M, et al. A natural killer-dendritic cell axis defines checkpoint therapy-responsive tumor microenvironments. Nat Med 2018;24:1178-91.

42. Kastenmüller W, Brandes M, Wang Z, Herz J, Egen JG, et al. Peripheral prepositioning and local CXCL9 chemokine-mediated guidance orchestrate rapid memory CD8+ T cell responses in the lymph node. Immunity 2013;38:502-13.

43. Spranger S, Dai D, Horton B, Gajewski TF. Tumor-residing Batf3 dendritic cells are required for effector T cell trafficking and adoptive T 
cell therapy. Cancer Cell 2017;31:711-23.e4.

44. Zhu AX, Finn RS, Edeline J, Cattan S, Ogasawara S, et al. Pembrolizumab in patients with advanced hepatocellular carcinoma previously treated with sorafenib (KEYNOTE-224): a non-randomised, open-label phase 2 trial. Lancet Oncol 2018;19:940-52.

45. El-khoueiry AB, Sangro B, Yau T, Crocenzi TS, Kudo M, et al. Nivolumab in patients with advanced hepatocellular carcinoma (CheckMate 040): an open-label, non-comparative, phase 1/2 dose escalation and expansion trial. Lancet 2017;389:2492-502.

46. Sia D, Jiao Y, Martinez-Quetglas I, Kuchuk O, Villacorta-Martin C, et al. Identification of an immune-specific class of hepatocellular carcinoma, based on molecular features. Gastroenterology 2017;153:812-26.

47. Harding JJ, Nandakumar S, Armenia J, Khalil DN, Albano M, et al. Prospective genotyping of hepatocellular carcinoma: clinical implications of next-generation sequencing for matching patients to targeted and immune therapies. Clin Cancer Res 2019;25:2116-26.

48. Ruiz de Galarreta M, Bresnahan E, Molina-Sánchez P, Lindblad KE, Maier B, et al. $\beta$-catenin activation promotes immune escape and resistance to anti-PD-1 therapy in hepatocellular carcinoma. Cancer Discov 2019;9:1124-41.

49. Di Tommaso L, Sangiovanni A, Borzio M, Park YN, Farinati F, et al. Advanced precancerous lesions in the liver. Best Pract Res Clin Gastroenterol 2013;27:269-84.

50. Grimm CF, Ortmann D, Mohr L, Michalak S, Krohne TU, et al. Mouse alpha-fetoprotein-specific DNA-based immunotherapy of hepatocellular carcinoma leads to tumor regression in mice. Gastroenterology 2000;119:1104-12.

51. Hong Y, Peng Y, Guo ZS, Guevara-Patino J, Pang J, et al. Epitope-optimized alpha-fetoprotein genetic vaccines prevent carcinogeninduced murine autochthonous hepatocellular carcinoma. Hepatology 2014;59:1448-58.

52. Geissler M, Mohr L, Weth R, Kohler G, Grimm CF, et al. Immunotherapy directed against alpha-fetoprotein results in autoimmune liver disease during liver regeneration in mice. Gastroenterology 2001;121:931-9.

53. Filmus J, Capurro M. Glypican-3: a marker and a therapeutic target in hepatocellular carcinoma. FEBS J 2013;280:2471-6. 